\title{
Design and Development of a Bipolar Power Supply for APS Storage Ring Correctors*
}

\author{
Y. G. Kang \\ Argonne National Laboratory, 9700 S. Cass Avenue, Argonne, II 60439
}

\section{Abstract}

The Advanced Photon Source (APS) requires a number of correction magnets [1]. Basically, two different types of bipolar power supplies (BPS) will be used for all the correction magnets. One requires dc correction only, and the other requires $\mathrm{dc}$ and ac correction. For the storage ring horizontal/vertical $(H / V)$ correctors, the BPS should be able to supply dc and ac current. This paper describes the design aspects and considerations for a bipolar power supply for the APS storage ring $\mathrm{H} / \mathrm{V}$ correctors.

\section{INTRODUCTION}

Basically, two different types of bipolar power supplies are used for all the APS correction magnets. One requires dc correction only, and the other requires dc and ac correction. For the storage ring $\mathrm{H} / \mathrm{V}$ correctors, the magnet current consists of a dc component and an ac component. Normally a power amplifier would be used for the BPS. Since the power amplifier type BPS uses the linear-mode power conversion which results in poor efficiency and large size, commercial BPSs are available for lower power levels. Therefore, it is desirable to design such a BPS using the switch-mode power conversion. However, due to the low pass filter effect of the vacuum chamber [2] uset in the storage ring, the amplitude of the ac component is substantially large, which makes designing a bipolar power supply using the switched-mode conversion techniques difficult. This paper deals with the design and development of a BPS using switch-mode power conversion for the APS storage ring $H / V$ correctors. A 150A BPS has been designed, prototyped, and tested, and about 650 units are being manufactured.

\section{SPECIFICATIONS}

The specifications for the BPS are given as follows:
a. Input dc bus voltage:
$70 \mathrm{~V}+10 \% /-15 \%$
b. Maximum output current:
* Maximum ac component:
* Correction frequency:
$150 \mathrm{~A}$ bipolar $=\mathrm{I}_{\mathrm{dc}}+\mathrm{I}_{\mathrm{ac}}$
75A(peak)@ 2.5Hz
* DC offset current:
$0 \sim 25 \mathrm{~Hz}$
$-150 \mathrm{~A} \sim+150 \mathrm{~A}$
c. Horizontal corrector:
Inductance: 6mH@ dc,
$3.25 \mathrm{mH} @ 25 \mathrm{~Hz}$
Resistance: $72 \mathrm{~m} \Omega @ \mathrm{dc}$, $137 \mathrm{~m} \Omega @ 25 \mathrm{~Hz}$
d. Vertical corrector:
Inductance: 7.6mH@ dc,
Resistance: $118 \mathrm{~m} \Omega @ \mathrm{dc}$,
e. Maximum output power:
$237 \mathrm{~m} \Omega @ 25 \mathrm{~Hz}$

*Work supported by the U.S. Department of Energy, Office of Basic En ergy Sciences under Contract No. W-31-109-ENG-38.

f. Ripple current:

g. Stability:

h. Reproducibility:

i. Tracking error:

j. Reference resolution:

\section{SYSTEM DESCRIPTION}

Figure 1 shows the simplified block diagram of a bipolar power supply. It consists of a power modulator (main power section), four Euro-cards for the regulator, digital-to-analog and analog-to-digital converters (DAC/ADC), converter and magnet monitoring \& interlocks, and a control power supply board. This BPS is water-cooled. The power supply control unit (PSCU) contains the intelligence that interfaces directly to each power supply hardware for control, menitoring, and communicating to the host computer. One PSCU can control as many as eight BPSs.

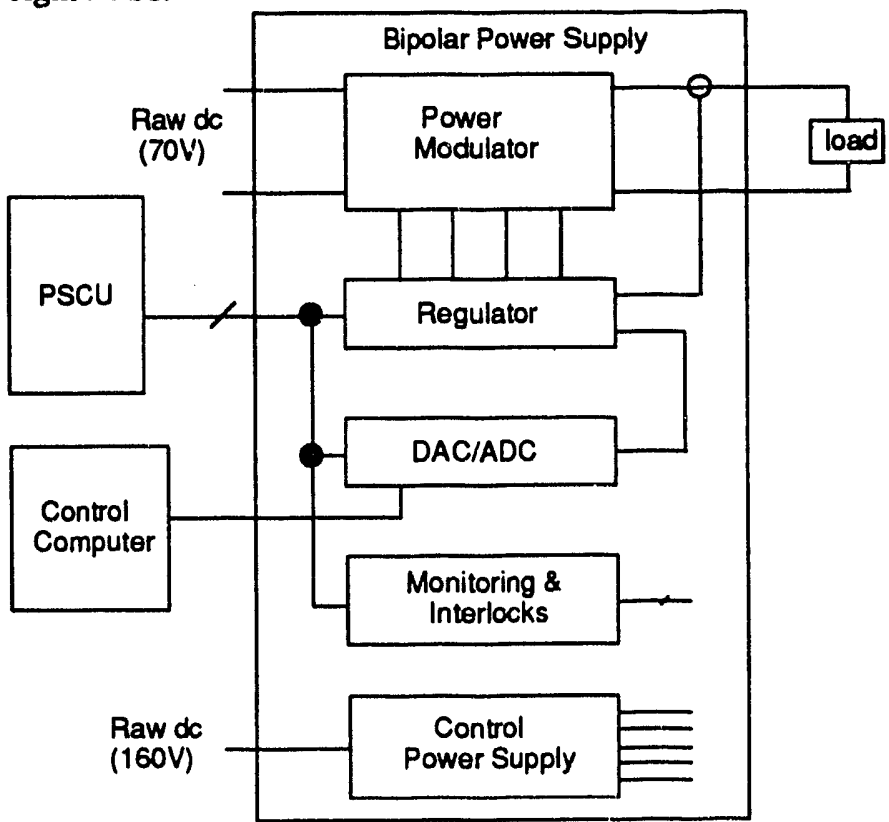

Figure 1: Simplified block diagram of the BPS.

Figure 2 shows the simplified circuit of the main power section. The topology is a full-bridge converter, and the switching frequency is $20 \mathrm{kHz}$ synchronized with the main clock. The regulation is achieved by controlling the pulse width. Insulated gate bipolar transistors (IGBTs) are considered for the switching devices due to their high current and voltage handling capability and easier drive requirement. Two water-cooled IGBT modules, Powerex IGBT type CM300DY-12H (dual package), are used for the switches. $L_{m}$ and $R_{m}$ represent the inductance and resistance of a correction magnet. For positive magnet current, switches $Q 1$ and $Q 4$ are closed. Only the upper switch, Q1, is controlled according to the pulse width modula-

\footnotetext{
The submitted manuscript has been authored

S. Government purposes.
} 


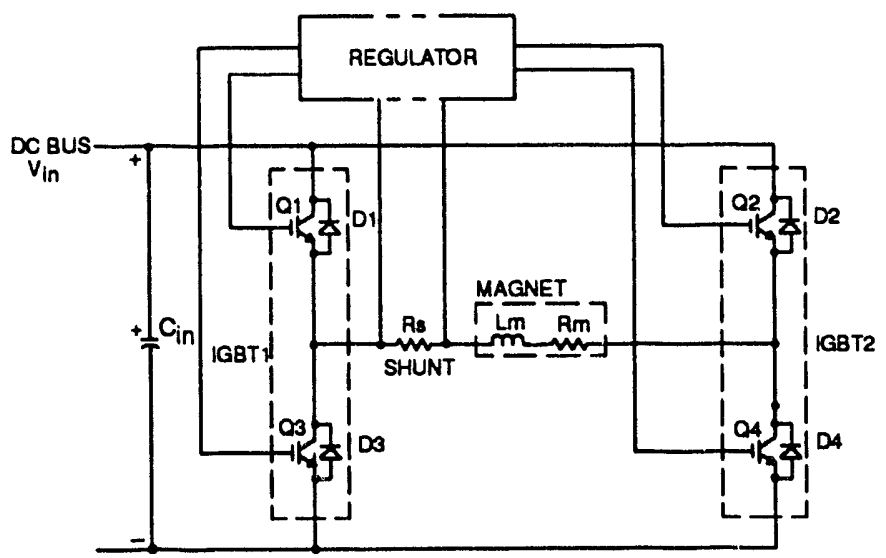

Figure 2. Simplified circuit of the main power section. tion (PWM) signal, while the bottom switch, Q4, remains closed. When Q1 is opened, the magnet current decays through the still-closed switch Q4 and diode D3. However, double gating, which controls both $\mathrm{Q} 1$ and $\mathrm{Q} 4$ simultaneously, is required for the negative di/dt. For negative current, switches Q2 and Q3 and diode D4 are used in a similar fashion. In order to regulate the magnet current, the magnet current information is fed back to an error amplifier input via a current measuring device and is compared with the reference voltage provided by a digital-toanalog converter (DAC). A $2 \mathrm{~m} \Omega$ water-cooled shunt resistor is used for the current measuring device. The unregulated dc input bus voltage, $V_{\text {in }}$, is provided by a separate three-phase ac/dc rectifier. The input dc bus, $V_{\text {in }}$, is determined by the following expression:

$$
V_{i n}=I_{p k}\left[\left(\omega L_{m}\right)^{2}+R_{m}^{2}\right]^{12} \sin (\omega t+\phi)+I_{d c} R_{m}
$$
where

$\mathrm{I}_{\mathrm{pk}}=$ peak value of the ac current, $\omega=2 \pi f(f=0 \sim 25 \mathrm{~Hz})$.

\section{CONTROL}

Figure 3 shows the simplified block diagram of a control scheme of correction magnet power supplies for the storage ring. The PSCU communicates to each power supply using op-

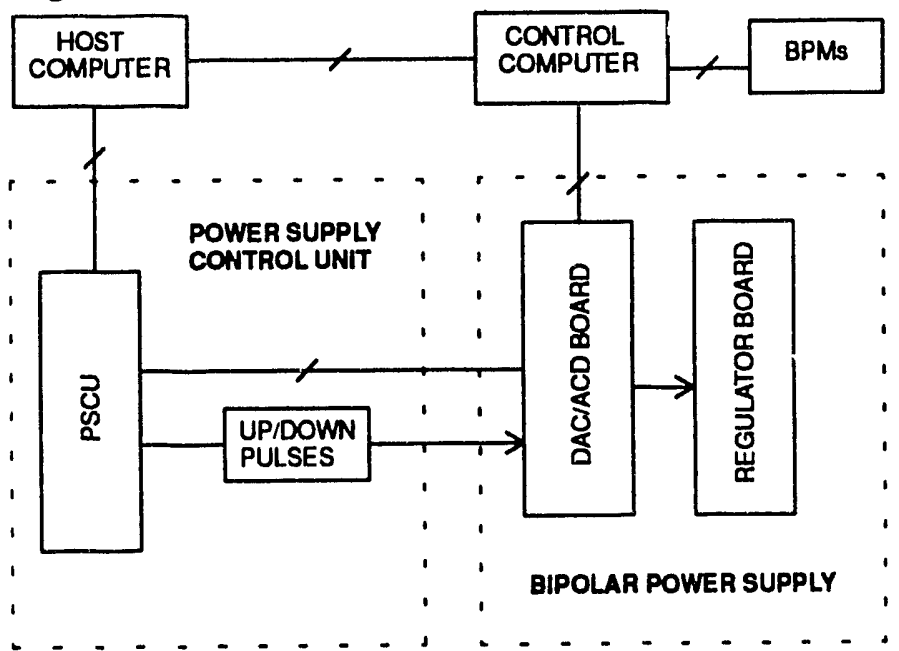

Figure 3. Simplified block diagram of a control scheme. tically coupled digital signals and differential shielded twisted pairs for analog signal transmission [3].

A 16-bit DAC is used for the reference signal generation. The host computer provides a current value to the PSCU and sends out a pulse train to the DAC to set the converter's output current. This current is the average (bias) correction current, and the current setting is done with an open loop. The control computer will sense the beam position using a number of beam position monitors (BPMs) and manipulate the information to compensate for the attenuation and phase delay due to the eddy current effect of the vacuum chamber. It then takes control from the host computer externally without passing through the PSCU and modulates the correction magnet's current by directly sending a pulse train in every $250 \mu$ s to the DAC board for the dynamic correction, which is done with a closed feedback loop.

\section{REGULATOR}

Figure 4 shows the simplified regulator circuit used to generate gating signals. The reference signal, $V_{n}$ from a DAC is 0 to $+10 \mathrm{~V}$ for the positive magnet current and 0 to $-10 \mathrm{~V}$ for the negative current. A polarity signal, which determines a set of switches to be controlled, is derived from the error voltage signal by using a comparator. A logic high signal, which selects switches Q1 and Q4, is obtained for a positive value. Similarly, a logic low signal, which selects switches Q2 and Q3, is obtained for a negative value. The feedback signal, $V_{b}$, is provided from a current measuring device. The voltage developed across a $2 \mathrm{~m} \Omega$ shunt resistor is amplified by an isolation amplifier to produce $+10 \mathrm{~V}(-10 \mathrm{~V})$ for maximum positive (negative) current. The output of the error amplifier, $V_{e}$, is compared with a $10 \mathrm{~V}$ ramp signal, $\mathrm{V}_{\mathrm{s}}$, at a comparator generating a pulse train. This PWM signal is combined with the polarity signal using an AND gate to determine which switch, Q1 or Q2, is to be controlled. The maximum duty cycle could be $100 \%$, including dc offset current and ac current.

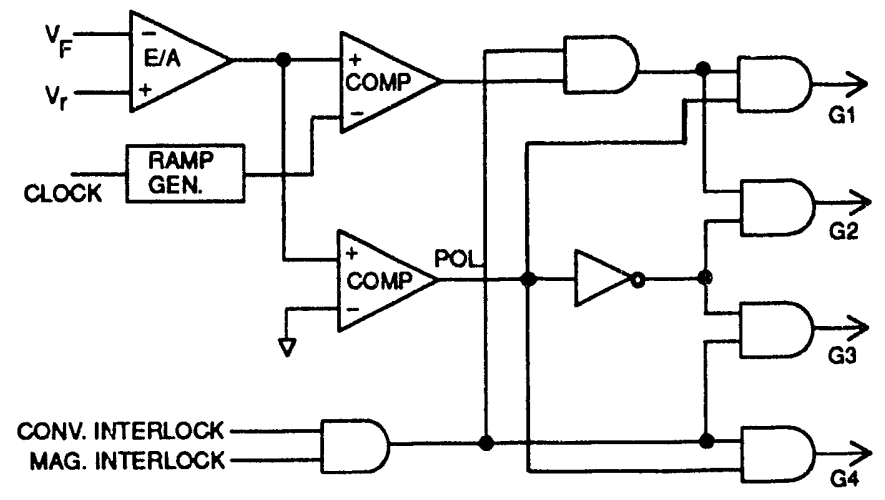

Figure 4. The simplified regulator circuit.

\section{DAC/ADC}

The reference signal for the regulator card is provided by a 16-bit DAC, and the magnet current information (feedback voltage) is converted to digital information by a 20 -bit $A D C$ to be used for monitoring. The DAC's input values are set by an input counter, which has three optically coupled input signals: one to reset it to zero for soft start, a second to increment it one 
bit at a time, and a third to decrement it one bit at a time. These input values are from the PSCU which also serially clocks out the 16 most significant bits from the DAC. For dynamic correction, the DAC is loaded directly by the control computer in a serial to parallel manner.

\section{MONITORING \& INTERLOCKS}

There are two Euro-cards provided in the BPS chassis for monitoring and interlocks, one for the converter (BPS) and the other for the magnet. The converter interlock card can monitor and interlock five temperatures for two IGBT modules, an input capacitor, retum water, and control power supply heat sink using PN junctions as temperature sensors over the range of 0 to $100 \mathrm{C}$ and three voltage signals for the magnet current, input bus, and control-power-supply failure. The magnet isiserlock card monitors and interlocks four temperatures for the magnet and its return water.

Each of the signals is compared to a fixed reference and the output of the comparator is used to set a flip/flop that latches the interlock until reset by a local or external reset pulse. All of these analog signals are buffered and output to the PSCU for monitoring. The analog signals for each interlock card are ORed, and the ORed output signals are sent out to the regulator vard. The ORed output signals from the converter and magnet interlock cards are combined by an AND gate to generate a shu: down signal. This causes the IGBT gate signals at the output of the regulator to be clamped, in case any of the failure signals activates.

\section{CONTROL POWER SUPPLY}

The control power supply card generates nine output voltages for the control circuitry as well as the IGBT driver circuitry. The topology used is a flyback converter with a feedback winding, which is used for line regulation and bias supply for the PWM IC, and the switching frequency is $80 \mathrm{kHz}$. Nine linear regulators are used for the load regulation. The input is fed from an output of the ac/dc unregulated rectifier, which also provides the dc bus for the main power section.

\section{TEST RESULTS}

A 150A BPS was prototyped and tested successfully. Figure 5 shows a measured magnet current waveform with its command signal, which is $25 \mathrm{~Hz}$ sinusoidal at $75 \mathrm{~A}$ (peak). Also, Figure 6 shows a measured magnet current waveform with its command signal, which is $25 \mathrm{~Hz}$ sinusoidal at $75 \mathrm{~A}$ (peak) plus $+75 \mathrm{~A}$ dc current. Note that the magnet currents follow their command signals.

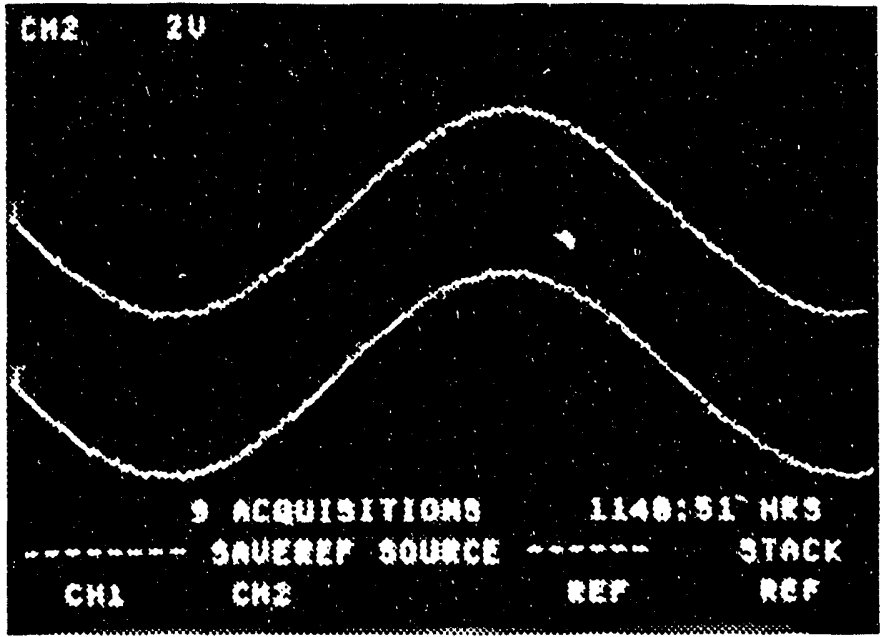

Figure 5: Measured magnet current with its command signal. Top: Magnet current Bottom: Command signal. $\left(I_{a c}=75 A_{p k}, \quad I_{d c}=0 A, f=25 \mathrm{~Hz}\right)$

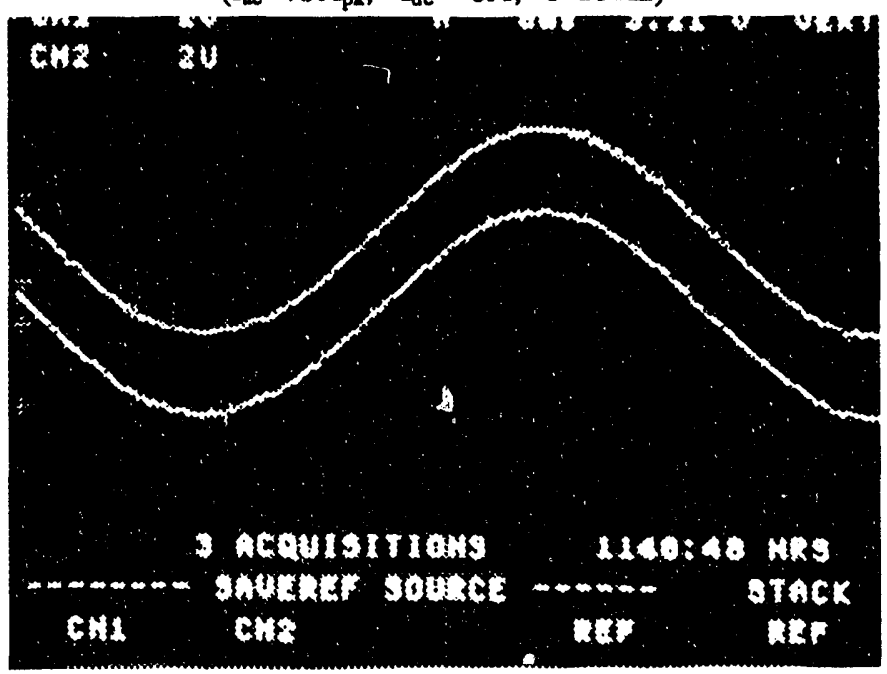

Figure 6: Measured magnet current with its command signal. Top: Magnet current Bottom: Command signal. $\left(I_{a c}=75 A_{p k}, \quad I_{d c}=+75 A, f=25 \mathrm{~Hz}\right)$

\section{REFERENCES}

[1] Y. G. Kang, "Correction Magnet Power Supplies for APS Machine," 1991 IEEE Particle Accelerator Conference Proceedings, pp. 911-913, 1991.

[2] W. Praeg, "Frequency Response of Storage Ring Magnets, Eddy Current Shielding of Vacuum Chamber," ANL LightSource Note LS-45, December 1985.

[3] O. D. Despe, C. Saunders, and D. G. McGhee, "Control Units for APS Power Supplies," these proceedings. 

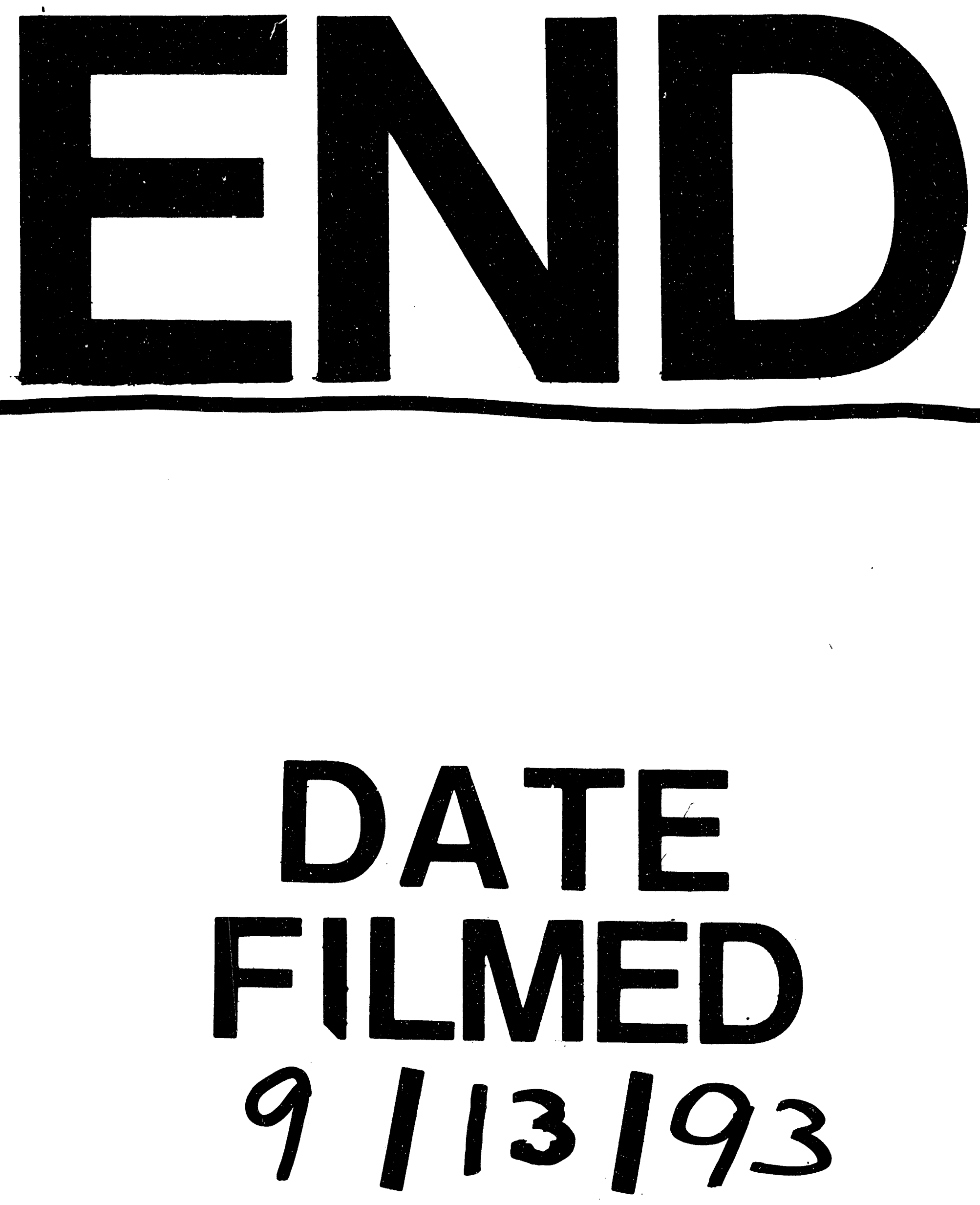
